\title{
Design and Manufacture of a Multiband Rectangular Spiral-Shaped Microstrip Antenna Using EM-Driven and Machine Learning
}

\author{
Ashrf Aoad \\ Department of Electrical and Electronics Engineering, Istanbul Sabahattin Zaim University, \\ Halkali Cad. No: 281 Halkali, Kucukcekmece, Istanbul, Turkey \\ esref.osmanlioglu@izu.edu.tr
}

\begin{abstract}
This paper presents a multiband rectangular microstrip antenna using spiral-shaped configurations. The antenna has been designed by combining two configurations of microstrip and spiral with consideration of careful selection of the substrate material, the dimension of the rectangular microstrip, the distance between the turned spiral, and the number of turns of the spiral. The efficiency and accuracy have been improved using machine learning algorithms as well. Machine learning has been studied to model the proposed antenna based on the performance requirements, which requires a sufficient training data to improve the accuracy. Three different machine learning models are applied to improve the accuracy and generalization performance and compared to simulation and measurement results. Simulation, measurement, and machine learning results confirm that the proposed antenna is a new electrically small and operating over a wide range of high-frequency bands between $1 \mathrm{GHz}-$ $4 \mathrm{GHz}$. Machine learning models have the best prediction ability with a mean square error (MSE) of 0.03 , and 0.05 . The antenna structure and size are compatible and suitable for several multi-band wireless mobile systems operating in Lband and S-band. The results, such as directivity, Half-Power Beamwidth, Voltage Standing Wave Ratio (VSWR), and Sparameter curves, are analysed and compared with the numerical formulation for both spiral and microstrip antennas.
\end{abstract}

Index Terms-Rectangular microstrip; Spiral; Machine learning; Error; Accuracy; Directivity.

\section{INTRODUCTION}

In recent years, the need for antennas have widely increased. It can certainly be considered as the main leading power behind the progressions being achieved in the field of modern communication and wireless technologies. Therefore, the interest in its development, production, and optimization appeared through various simulation techniques. The methodology discussed in this paper can be categorized into three categories: designing the proposed antenna by combining two types of antennas, fabrication, and modeling by artificial intelligence [1].

Because of the attractive similarity between the properties of microstrip patch and spiral antennas, they have been greatly applied in wireless communications, biological medicine, radar and electronic counter measurements [2],

Manuscript received 2 September, 2020; accepted 11 January, 2021.
[3]. They are more useable among other antennas and have attractive configurations for researchers and users due to their lightweight, low profile, cost, implementation, and ease for combining qualitative to obtain new configuration and performance [4]. Therefore, these antennas can be easily manufactured in large quantities. Many shapes and types of antennas based on different design processes and reconfiguration techniques have been researched. It has been noticed that microstrip and spiral antennas have simple and same condition of the configuration, consisting of a very thin radiating element $t<<\lambda_{o}$ (where $\lambda_{o}$ is the freespace wavelength and $t$ is the thickness) on a side of a substrate material (usually in the range of $2.2 \leq \varepsilon_{r} \leq 10$, where $\varepsilon_{r}$ is permittivity constant), while the ground plane is on the other side [5]. Selection suitable substrate material and configuration seem to offer possibilities for reducing the size and keeping the performance of an antenna. The radiating elements are usually photo-etched on the dielectric substrate, while the feed lines are laid on or passed through the dielectric. Both the permittivity and the thickness of the substrate material influence the performance. Typically, the form of radiating element of microstrip antennas may be circular, square, rectangular, thin patch, elliptical, triangular, and/or any different configuration [4], [5]. In the spiral antennas, it may be single, double or more windings directed right or left with different configurations, which are logarithmic, planar circular, rectangular, selfcomplementary, and Archimedean spirals [6]. In addition, both of the two types of the proposed may be electrically small and an element of a set of an array [7]-[9]. Generally, the number of antennas in an array can be as small as two or larger (several hundreds).

The spiral antennas are referred to as frequency independent antennas. Antenna polarization is an important condition when researching and designing antennas [10]. Polarization is one of the fundamental characteristics of the antenna and in demand for many applications. Microstrip and spiral antennas are circularly polarized [5]. Circular polarization can be either right-handed circular polarization (RHCP) or left-handed circular polarization (LHCP), depending on the direction of the rotation of the field propagation versus time [11]. The input impedance $\left(Z_{\text {in }}\right)$ is 
based on the type (strip or coaxial elements), dimension (thickness of the substrate and dielectric constant), and configuration (shape and physical elements) of the feeding system of the antenna. Microstrip and spiral antennas can be generally designed to transform $Z_{\text {in }}$ to 50 and $188 \mathrm{Ohms}$, respectively [5].

As proven above, the similarity of characteristics of microstrip and spiral antennas makes them attractive and possible to combine and present as a novel configuration [4].

As is well known, the modeling of an antenna is permanently achieved in 3D electromagnetic simulation environment, such as Applied Wave Research (AWR), High Frequency Structure Simulator (HFSS), Advanced Design System (ADS), Computer Simulation Technology (CST), etc., while each of them has a different computational method. Beside 3D electromagnetic simulation environment, the machine learning (ML) has been identified as competitive intelligence technique for antenna modeling and optimizing [1], and it can be widely utilized in several disciplines, such as engineering, education, science, meteorology, medicine, human resources recruiters, banking and economics. Various ML algorithms have been introduced to model characteristics of antennas, such as gain, directivity, and S-parameters $\left(S_{11}, S_{22}\right.$, etc. ) [1]. They are mathematical processes performing random calculation during learning process and have the capability of learning and generalization to improve the antenna modeling and synthesis efficiency. Therefore, using ML models for antenna modeling can improve the efficiency and accuracy of the antenna. ML models are trained to realize the mapping between the input and output vectors to obtain the prediction/result as a data set. The training process finds out parameters that predict the best model for presented data. Several ML methods have been applied for antenna modeling and synthesis, such as Gaussian process [12], support vector machine [13], artificial neural networks (ANNs) [14], [15], and space mapping [16]. In [17], the performance of selection operator (lasso), ANNs, and knearest neighbour ( $\mathrm{kNN}$ ) ML is investigated for designing and optimizing of double T-shaped monopole Antenna. In [18], multistage collaborative ML (MS-CoML) methods, such as single-output Gaussian process regression (SOGPR) and symmetric multi-output Gaussian process regression (MOGPR) methods are introduced to collaboratively construct extremely accurate multi-task surrogate solutions/models for different antennas. They are single band microstrip antenna, substrate integrated waveguide (SIW) cavity-backed slot antenna (CBSA), and tri-band patch antenna. Therefore, for antenna modeling, ML algorithm can be classified as a constructor of a surrogate model/solution and as an optimization method [1].

In this study, three ML regressions have been used, which are decision tree regression (DTR), decision forest regression (DFR), and artificial neural networks (ANNs) [19]. These regression algorithms are popular in several applications. They save simulation time, train fast, and perform successfully, as well as are considered as a powerful tool to the overfitting problem.

The main contribution of this work is developing a novel configuration to obtain an electrically small lateral size antenna, multiple operating frequencies to fulfil the coverage of $1 \mathrm{GHz}-4 \mathrm{GHz}$ and return losses with the directivity (D) of $7 \mathrm{dBi}$, a Half Power Beam Width (HPBW) lower than $90^{\circ}$, and good propagation characteristics while maintaining matching of VSWR $\leq 2$. ML algorithms are applied to predict new models, as well as to calculate various metrics used for measuring the model's performance, such as the mean squared error $(M S E)$, root of mean squared error ( $R M S E)$, relative absolute error $(R A E)$, and relative squared error $(R S E)$. Finally, the accuracy and generalization for predicted, simulated, and measured models are compared, as well as differences and agreements between the obtained models have been cleared up.

\section{Antenna Design Stages Permissions}

To achieve the objective previously mentioned, several configuration steps have been experimentally realized and numerically evaluated [4]. The proposed antenna consists of three parts: radiator (microstrip patch: rectangular and spiral), substrate, and ground plane. The radiator and the ground plane are separated by the substrate. Each part has different thickness, while the length and the width of the substrate and ground planes have the equal lateral dimension [14]. The radiator part consists of a very thin metallic strip placed on the substrate. Thickness of the radiator is $t_{r}=0.0158 \mathrm{~cm}=0.0009 \lambda_{o}$, where $t_{r}<<\lambda_{o}$ and the tangential electric field across a metal is zero $\left(E_{t}=0\right)$. Copper is the most heavily evaluated and used metal because of its electrical characteristics. It has a very high conductivity and is recognized as a Perfect Electric Conductor (PEC). For practical applications, most metals, such as steel, aluminum, gold, and copper can be considered as PECs. The microwave substrate is Rogers RT 5880 (lossy) of dielectric constant $\varepsilon_{r}$ of 2.2, thickness of the height $h$ of $0.1588 \mathrm{~cm}=0.008 \lambda_{o}$, where $h<<\lambda_{o}$, and the tangent loss $\delta$ of 0.0009 . The ground plane is necessary in the proposed application. It is an electrically conductive plane and has different thickness than the conductor. The ground plane is a reflector to reflect the electromagnetic radiation. The radiation process is due to the fringing field between the periphery of the microstrip and the ground plane. The feeding system is coaxial located at the center $(0$, $0,0)$ of the rectangular patch and adjusted for optimal matching of $50 \mathrm{Ohms}$ of characteristic impedance. Typically, the matching process is performed by controlling the inner, outer radius of the feeding system and the length of the slot (cylinder). The characteristics of the feeding system and the substrate (except the width $W_{s}$ and length $L_{s}$ of the substrate) will be constant through experimental (configuration) steps. Each experimental step leads to another with considering the undesirable mutual coupling [20].

\section{A. Microstrip Patch Antenna}

As is shown in Fig. 1, the length and width of the basic rectangular microstrip patch antenna are $0.3 \mathrm{~cm}$ and $0.5 \mathrm{~cm}$, respectively, without adding any windings of the spiral. 
Note that the conductor/radiator and the feeding system are photo-etched on the thin dielectric substrate. For this configuration, both transmission line and cavity models are most accurate and can be easily analysed [21], [22].

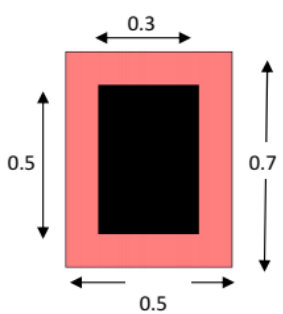

(a)

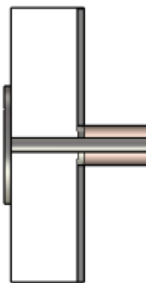

(b)
Fig. 1. (a) Rectangular patch antenna \& (b) side view.

For an efficient conductor, (1) is used to calculate the resonating frequency for rectangular microstrip antennas as shown in Fig. 1 [23]. Rectangular microstrips are preferred due to easy calculation, reconfiguration, and modeling.

$$
f_{o}=\frac{c}{2 W \sqrt{\varepsilon_{r}+1 / 2}}
$$

where $W$ is the width of the rectangular patch and $c$ is the speed of light in free space. The simulation of Fig. 1 leads to the result of $f_{0}=2.5287 \mathrm{GHz}$ and $13.927 \mathrm{GHz}$, while calculating (1) numerically leads to the result of $f_{0}=$ $39.5 \mathrm{GHz}$. There is a huge difference between simulated $(1 \mathrm{GHz}-4 \mathrm{GHz})$ and calculated results of $f_{0}$, which direct us to other reconfigurations.

\section{B. Second Configuration}

This case is realized by adding a spiralled rectangular arm with a rotation of $360^{\circ}$ in the direction of counterclockwise $(\mathrm{CCW})$ starting from the left edge at distance of $(-0.5,0.25$, $0.0158)$ and ending at $(-1.0,-1.25,0.0158)$ (all dimensions are in $\mathrm{cm}$ ) as shown in Fig. 2. The arm is fed by the basic rectangular microstrip shown in first case. The width of the spiral rectangular arm is $0.25 \mathrm{~cm}$, and the empty area is $0.5 \mathrm{~cm}$. It is noted that the length $L_{s}$ and the width $W_{s}$ of the substrate are increased around $271 \%$ and $360 \%$, respectively.

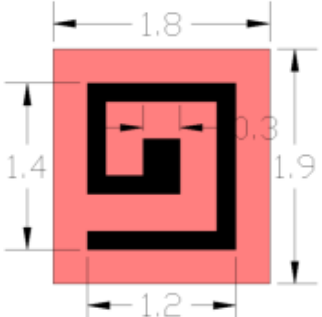

Fig. 2. Rectangular patch with one added spiralled arm.

Figure 2 shows new configuration of the combining the rectangular microstrip and spiral antennas. Using (1) leads to the result of $9.88 \mathrm{GHz}$, which is approximately close to one of the simulated results not shown in Fig. 4. The antenna is still operating more efficiently outside the range of $1 \mathrm{GHz}-4 \mathrm{GHz}$ range.

\section{Third Configuration}

Third case is similar to the previous case by adding another spiral rectangular arm, and under the same physical conditions. The new configuration and its increased dimensions are shown in Fig. 3.

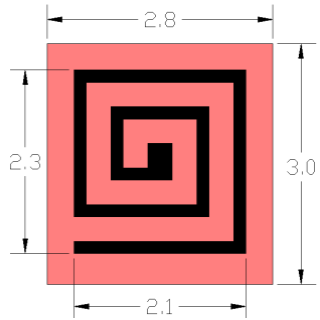

Fig. 3. Rectangular patch with two added spiralled arms.

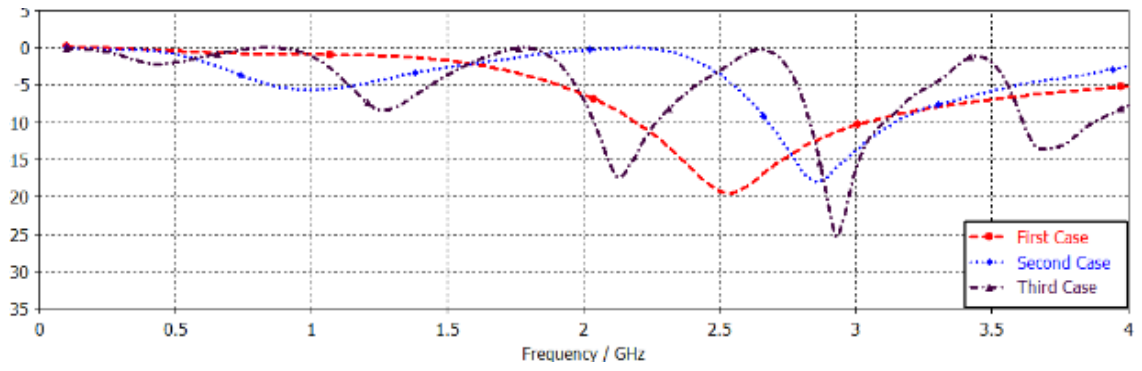

Fig. 4. $S_{11}$ for 3 simulation configurations.

Figure 4 shows the positive effect on the performance of the antenna when adding new spiral arms gradually as new frequency bands $(1 \mathrm{GHz}-4 \mathrm{GHz})$ appeared. Consequently, the new spiral arm can be easily added, and then the antenna is simulated again to demonstrate the potential for obtaining new frequency bands as shown in Table I.

TABLE I. RESULTS OF THE THIRD SIMULATION CASE OF RSMA.

\begin{tabular}{|c|c|c|c|c|}
\hline $\boldsymbol{f}_{\mathbf{0}}(\mathbf{G H z})$ & $\boldsymbol{S}_{\mathbf{1 1}}(\mathbf{d B})$ & $\mathbf{D}(\mathbf{d B i})$ & VSWR & $\begin{array}{c}\text { HPBW } \\
\boldsymbol{\varnothing = 9 0}\end{array}$ \\
\hline 1.2658 & -7.887 & worse & worse & worse \\
\hline 2.1213 & -17.11 & 5.477 & 1.324 & $63.1^{\circ}$ \\
\hline 2.8927 & -25.45 & 7.026 & 1.112 & $44.1^{\circ}$ \\
\hline
\end{tabular}

\section{Fourth Configuration}

This final simulation case shows the final configuration of the proposed antenna. In other words, the configuration form is a rectangular spiral-shaped microstrip antenna (RSMA) as shown in Fig. 5. The spiral arm is reduplicated as a length of transmission lines of characteristic impedance [22]. Each step of the reduplication increases the spiral arm by $0.5 \mathrm{~cm}\left(0.00095 \lambda_{o}\right)$. The empty spaces between spiral arms and mid microstrip are not changed and have a width of $0.5 \mathrm{~cm}$. While the distance between angular arms is increasing constantly, the size of the shape of RSMA also increases the width and the length that affected the results 
positively. The curvature between arms is at $\pi / 2$ radians counterclockwise. The proposed antenna can be defined by its height, width, and number of horizontal and vertical spiral turns. This will determine the limit of lower and upper frequency bands range (see the final simulation and measured results in figures of Section III). The geometrical parameters of the antenna are optimized using commercial CST Microwave Studio based on the finite integration technique (FIT).

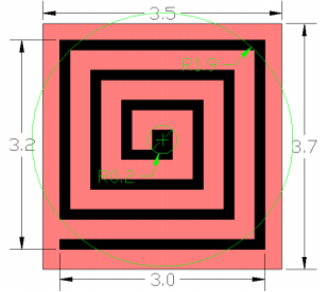

Fig. 5. Final model of the rectangular patch with three added spiralled arms.

The reduction of mutual coupling is considered the most important process in the case of design both single and arrays of rectangular, circular spirals and microstrips [24] [25], [26]. Mutual coupling, in the case of voltage/power transmitted, is confused by possible mismatches at both channels of transmitter and receiver. Therefore, the empty distance between arms, widths, and thickness of the configuration are carefully experimented and located. The wavelength is larger than the total length of the spiral. So, the magnitude and the current scattered constantly through the surface of the proposed conductors. The current path starts distributing from the mid to spiral conductor in a clockwise direction. The distribution of the current through the conductors makes the radiation [27].

There are 13 straight segments/sections (total of the conductor patches of the spiral) that show the negative and positive mutual inductance paths (see Fig. 6). Figure 6 shows that opposite segments carrying current in the inverse direction have negative mutual inductance while having positive mutual inductance in the case of the same directions [3], [28], [29]. The identity of all segments is assumed for the magnitude and the phase of the current.

Therefore, 13 segments and 3.25-spiral turns can be written as a total of all inductances added to (positive negative) mutual inductances and mathematically realized as

$$
c=\sum_{i=1}^{m} c_{i}+2\left[\sum_{j=1}^{n}\left(\sum_{i=1}^{m-4} M_{i, i+4 j}-\sum_{i=1}^{m-2} M_{i, i+2 j}\right)\right],
$$

where $m=13$ is the number of segments, $n=3$ is the number of whole spiral turns, and $M$ is the total of positive and negative mutual inductances. Similarly, the negative and positive mutual inductances $M^{ \pm}$can be given by

$$
M^{+}=4[n(n-1)]+2 n[m-4 n] .
$$

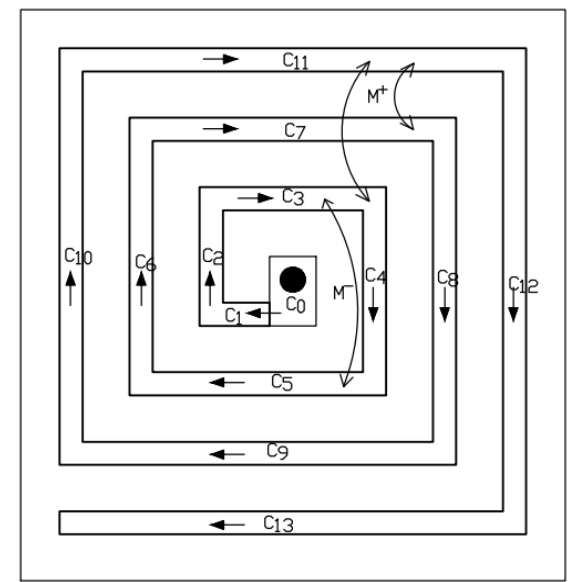

Fig. 6. Positive and negative mutual inductance paths.

The expression of $M^{-}$is

$$
\begin{gathered}
M^{-}=4 n^{2}+ \\
+2 n(m-4 n)+(m-4 n-2)(m-4 n-1)[(m-4 n) / 3] .
\end{gathered}
$$

For all that $\mathrm{M}^{-}$is larger than $\mathrm{M}^{+}$, so their contribution ratio to all inductances $(C)$ values is much less due to the much considerable distance.

Figure 7 implies that the antenna radiates best at $1.6 \mathrm{GHz}$, $2.04 \mathrm{GHz}, 2.4 \mathrm{GHz}$, and $2.9 \mathrm{GHz}$ with a bandwidth of $0.056 \mathrm{GHz}, 0.105 \mathrm{GHz}$, and $0.145 \mathrm{GHz}$, respectively, at the return loss of $-10 \mathrm{~dB}$. Besides, VSWR curve in the frequency bands is presented in Fig. 8 .

For $f_{\text {low }}$ and $f_{\text {high }}$, an additional numerical calculation can be verified. Enveloping two circles on the mid and the outer spiral arm with radius of $R_{i n}=0.2 \mathrm{~cm}$ and $R_{\text {out }}=1.9 \mathrm{~cm}$ as shown in Fig. 5. Note that the small circle must touch the upper edges of the middle microstrip with a circumference of $1.33 \mathrm{~cm}$, and the large circle must also touch the inner edges of the outer arm of the spiral with a circumference of $11.99 \mathrm{~cm}$.

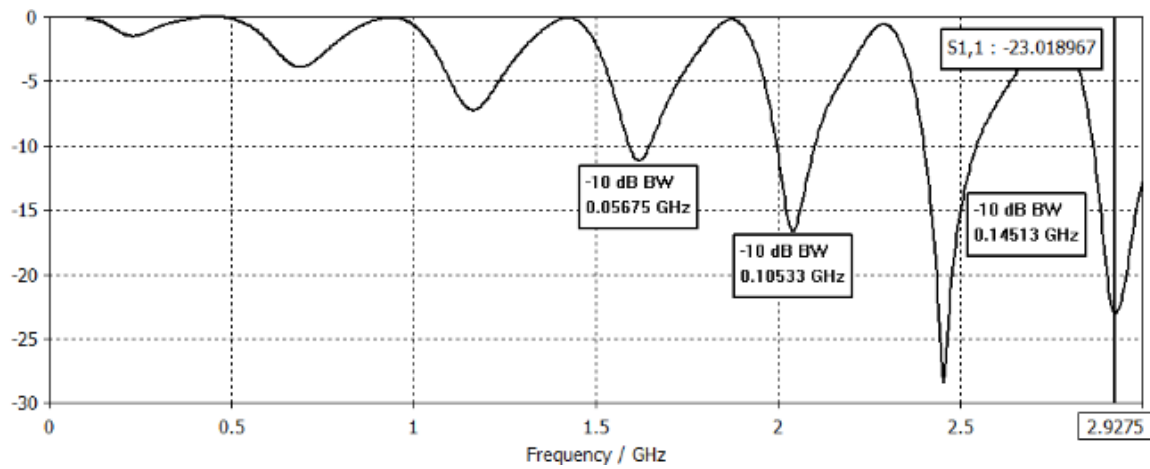

Fig. 7. $S_{11}$ for the final simulation case. 


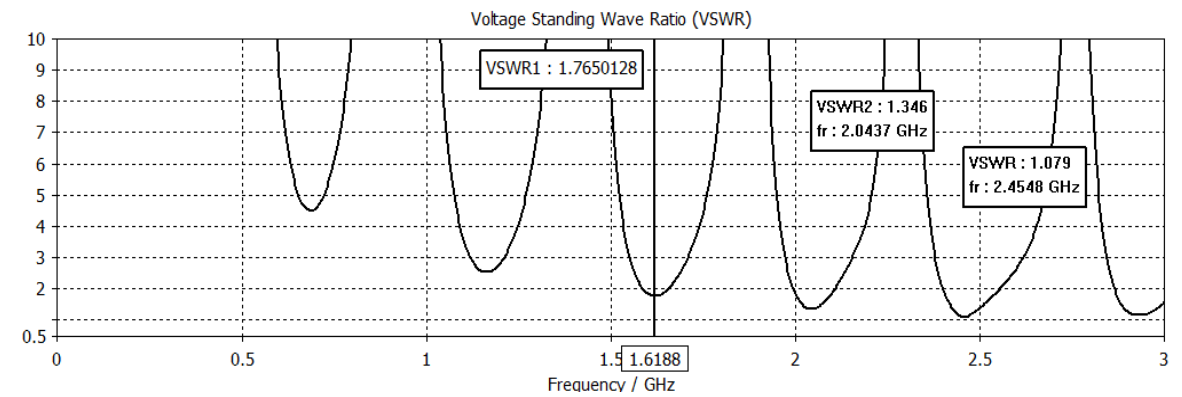

Fig. 8. VSWR result for the final case.

For low and high frequencies, the spiral formulas are given as:

$$
\begin{aligned}
& f_{\text {low }}=\frac{c}{2 \pi R_{\text {out }}}, \\
& f_{\text {high }}=\frac{c}{2 \pi R_{\text {in }}} .
\end{aligned}
$$

According to (5) and (6), the result of operating frequencies is $2.51 \mathrm{GHz}$ and $23.87 \mathrm{GHz}$ for $f_{\text {low }}$ and $f_{\text {high }}$, respectively.

Comparing the results shown in Table II to the previous results shown in Table I, the performance of the proposed antenna has been clearly realized by adding the final spiral arm.

\begin{tabular}{|c|c|c|c|c|c|}
\hline \multirow{2}{*}{$\begin{array}{c}f_{0} \\
(\mathbf{G H z})\end{array}$} & \multirow{2}{*}{$\begin{array}{l}S_{11} \\
(\mathrm{~dB})\end{array}$} & \multirow{2}{*}{$\underset{\text { (dBi) }}{D}$} & \multirow{2}{*}{ VSWR } & \multicolumn{2}{|c|}{ HPBW } \\
\hline & & & & $\emptyset=\mathbf{0}^{\circ}$ & $\emptyset=90^{\circ}$ \\
\hline 1.618 & -11.16 & 2.75 & 1.765 & $48^{\circ}$ & $52.5^{\circ}$ \\
\hline 2.043 & -16.65 & 7.01 & 1.346 & $42.9^{\circ}$ & $43.6^{\circ}$ \\
\hline 2.454 & -28.37 & 7.38 & 1.079 & $45.1^{\circ}$ & $39.5^{\circ}$ \\
\hline
\end{tabular}

TABLE II. RESULTS OF THE FINAL SIMULATION CASE OF RSMA.

Now, the results proven below characterize the far-field radiation pattern in the positive z-direction of the final configuration in addition to the directivity over phi $\varnothing$ and theta $\theta$ angle in linear scaling mode (Fig. 9 and Fig. 10).

The results are shown for successive simulation cases. The final simulation case is considered as the optimum, which demonstrates the aim of the designed antenna; hence, it can be summarized as small size, narrow beam, and low frequency. In the third simulation case, some good and acceptable results have been obtained as well. However, entirely acceptable results that maintain the objective of the proposed antenna were obtained in the final case.
Directivity Abs (Phi=0)

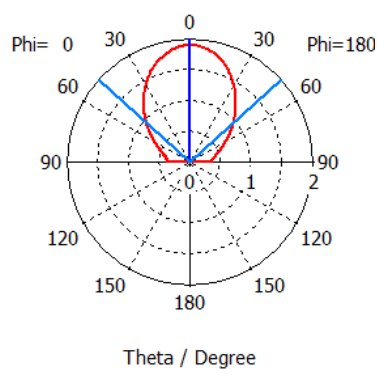

(a)
Directivity Abs (Phi=0)

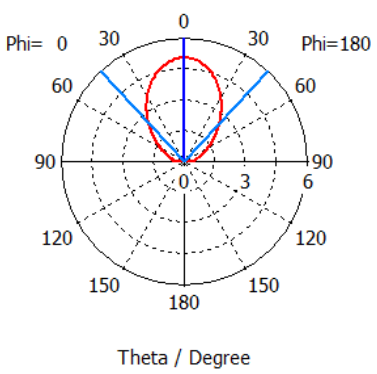

(b)
Directivity Abs (Phi=0)

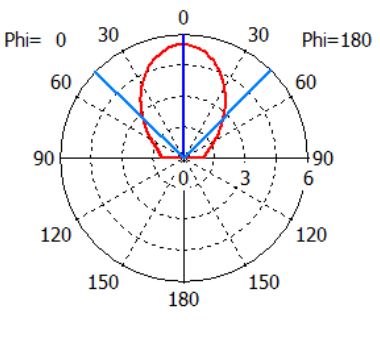

Theta / Degree

(c)

Fig. 9. Two-dimensional far-field patterns at $\emptyset=0^{\circ}$ : (a) $f_{0}=1.618 \mathrm{GHz}$; (b) $f_{0}=2.043 \mathrm{GHz}$; (c) $f_{0}=2.454 \mathrm{GHz}$.

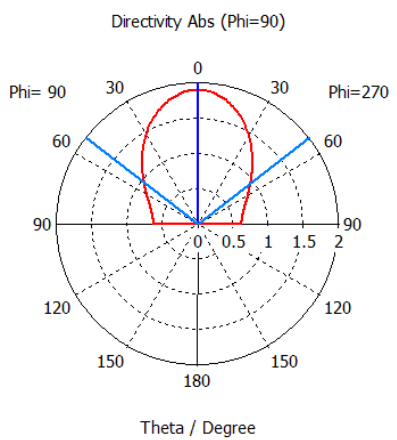

(a)

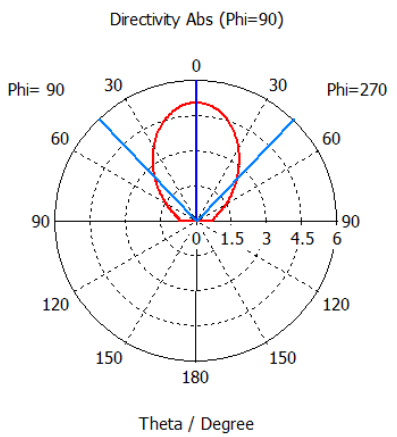

(b)

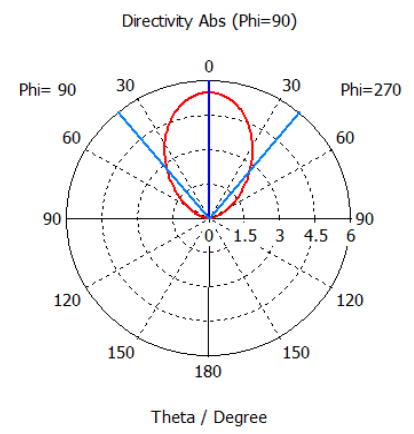

(c)

Fig. 10. Two-dimensional far-field patterns at $\emptyset=90^{\circ}$ : (a) $f_{0}=1.618 \mathrm{GHz}$; (b) $f_{0}=2.043 \mathrm{GHz}$; (c) $f_{0}=2.454 \mathrm{GHz}$.

\section{MEASUREMENT}

Figure 11 shows the front section of the fabricated model, the metal part is copper (PEC) and spirally curled on the substrate. The back section is fully covered $S_{11}$ by the copper (PEC) which is called ground, drilled in the midpoint of radius $0.2 \mathrm{~cm}$ as an outer cylinder, but the inner cylinder is about $0.0585 \mathrm{~cm}$ to allow access of feeding. Middle section that has a dielectric constant of 2.2 is called substrate with material of Rogers RT 5880. 


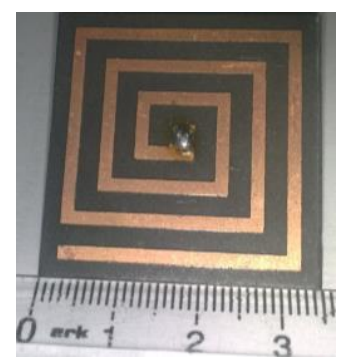

Fig. 11. Front section of fabricated model of RSMA.
Figure 12 clearly shows the disagreement between simulation and measurement models. Hence, ML regressions have been used to obtain an equivalent/surrogate model using the simulation model as an input included to the training data of the ML (see equations in Section V).

Figure 13 shows the frequency chart describing the change of resonant frequencies while moving from a simulation case to the next in addition to the measurement case at the end.

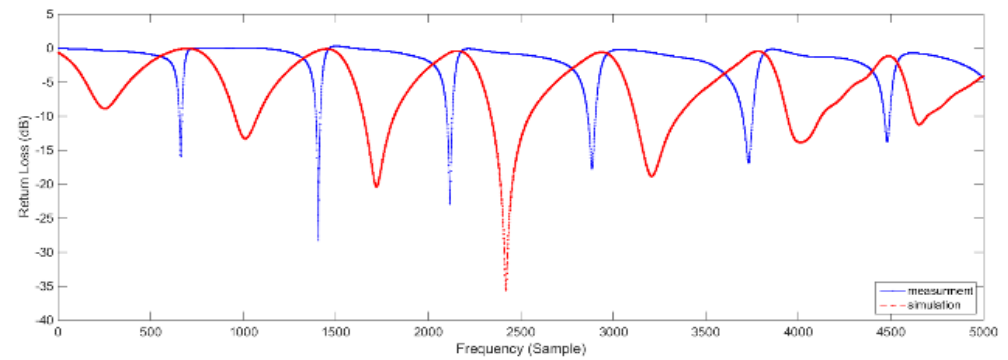

Fig. 12. Comparison of $S_{11}$ of simulation and measurement models.

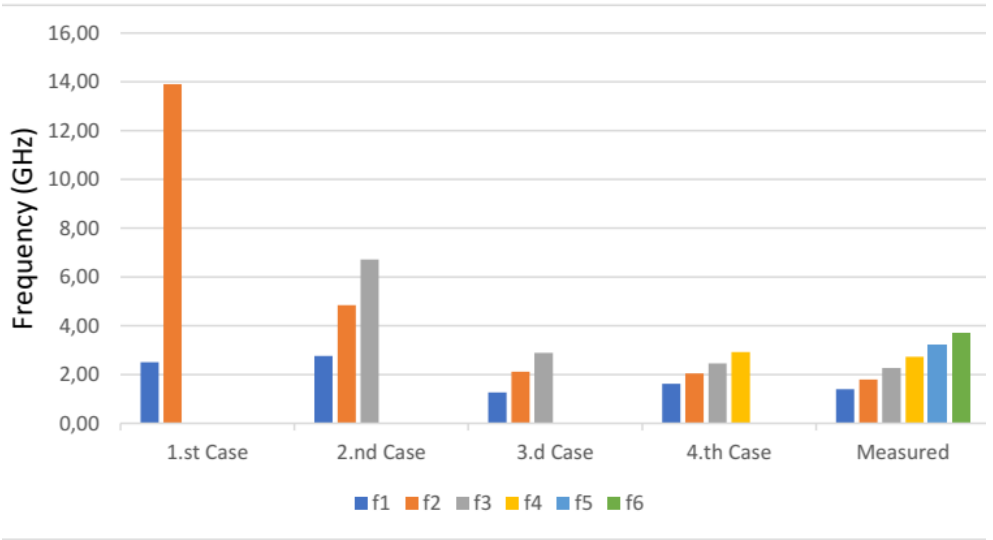

Fig. 13. Chart of operating frequencies for all simulation and measurement cases.

\section{MACHINE LEARning REgRession AlgorithMS}

The goal of using ML algorithms in antenna modeling is to predict new models' characteristics using the training data generated by the original computational EM model [1], [30]. In other words, ML is used to evaluate model's accuracy and generalization [1], [18]. It can be realized by learning the interconnection between the input $x$ and the corresponding output $y$ parameters by fitting a model from the data such that

$$
y=M(x),
$$

where $y$ is the output of the antenna model and $y \in Y \subset \mathbb{R}^{u}$, while $u$ is the output variable and $x \in X \subset \mathbb{R}^{c}$ is an input vector collecting $c$ modeling variables. Therefore, the parameters of the models are typically computed to reach the minimized prediction error. The prediction error is the difference between the original (measured) value and the predicted value. Mean squared error (MSE) function is used to measure the accuracy while the MSE value settles at the minimum

$$
M S E=\frac{1}{n} \sum_{i=1}^{n}\left(y_{i}^{\text {pred }}-y_{i}^{\text {meas }}\right)^{2},
$$

where $y_{i}^{\text {pred }}$ is the predicted value, $y_{i}^{\text {meas }}$ is the measured value, and $n$ is the number of data samples, starting from $i$ sample. Equation (9) generally defines a fundamental performance metric that can be written as

$$
\mathbb{M}=\mathbb{G}_{i=1, n}^{z}\left[\mathbb{N}^{z} \mathbb{D}^{z} y_{i}^{\text {pred }}, y_{i}^{\text {meas }}\right] \text {, }
$$

where $z$ is numerical index of the regression methods, $\mathbb{G}$ is the process of aggregation of sample distances through data, $\mathbb{D}$ is the process of determining sample distance, and $\mathbb{N}$ is the process of normalization. According to Table III, performance metrics can be used for ML regressions, which are root of mean squared error (RMSE) that measures the average of the squares of the error, and then applies the square root to the obtained result, relative absolute error $(R A E)$ that is the percentage of the result error, and relative squared error $(R S E)$ that normalizes the total squared error by dividing the total error of the predicted values.

Three ML regression algorithms are used for the purpose of antenna modeling and comparison of results: decision tree regression (DTR), decision forest regression (DFR), and artificial neural networks (ANNs) [19]. In general, the regression algorithm learns the value of the parameters of a function for an exceptional model of data. It might predict 
antenna performance's height by using a height function or predict the probability of performance drop based on test data values [31]. Regression algorithms have an advantage represented in combining input parameters from various characteristics by setting the contribution of each characteristic of the data to the regression function. The models of the antenna are built and trained using Azure machine learning based on global infrastructure that is made up of physical and connective network elements. The physical element consists of more than 160 Azure datacenters interconnected with one of the greatest networks on the world. These datacenters provide high availability, minimal latency time, scalability, and the latest advancements in cloud infrastructure [32], [33].

\section{A. Artificial Neural Networks (ANNs)}

ANNs are the most common method to calculate and develop nonlinear regression based on a model of biological neurons [14]. ANN is the structure of many layers. These layers are categorized as follows: input layer, hidden layer, and output layer; each layer contains neurons. Neurons are interconnected with the corresponding links (weights). They basically perform computations, and then transmit knowledge from the input to the output. Multilayer ANN model is trained and defined as

$$
x_{j}^{k+1}=f\left(\sum_{d} W_{d j}^{k} x_{d}^{k}+B_{b j}^{k}\right),
$$

where $W_{d j}^{k}$ are weights connecting $d^{\text {th }}$ neuron in the layer $k$ to $j^{\text {th }}$ neuron in the layer $k+1$; they were initialized randomly. $B_{b j}^{k}$ represents the bias of $j^{\text {th }}$ neuron in the layer $k$, and $f($.$) term represents the nonlinear activation$ function, such as the sigmoid function.

\section{B. Decision Tree Regression (DTR)}

Decision tree is regression or classification model built in the form of a tree and also known as a predictive model [34]. It is a stepwise method, depending on using a predefined loss function $L y, F x$ to optimize the parameter values in the model. In other words, it measures the error in each learning stepwise, and then minimize/correct it in the following step, which is continued to the number of iterations of $M$ [30].

In general, the decision tree splits a huge amount of training data into smaller subset training datasets and features containing instances with similar values (homogenous), and an associated decision tree is incrementally optimized [35]. The result is a tree with two kinds of nodes, such as decision and leaf/terminal nodes. In addition, from two or more kinds of branches extending from a decision node, each represents values for the parameters that are tested. Leaf node is considered as a decision on the numerical target output. Knowing that decision trees can handle and generate a model with two kinds of data, which are categorical and numerical data. The root node is the topmost decision node in a tree corresponding to the bestead model predictor as shown in Fig. 14. The size of the tree depends on the size of the input and output data $x_{i}, y_{i}{ }_{i=1}^{n}$. The aim is to achieve an estimated approximation of $\hat{F}(x)$ to a function of $F(x)$, which reduces the expected value of some values of the loss function

$$
\hat{F}(x)=\arg _{F} \min \mathrm{E}_{x, y} \mathcal{L}(y, F(x)) .
$$

Most important part of algorithm for building decision trees is ID3 [36]. Therefore, ID3 is a realization algorithm developed to construct a decision tree for regression by replacing Gain with standard deviation reduction (SDR).

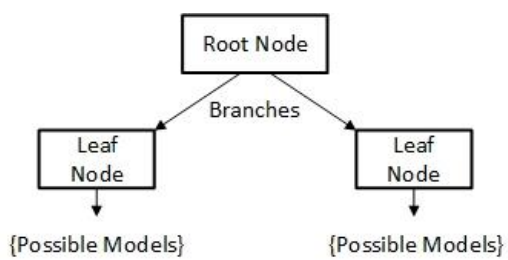

Fig. 14. Perspective of decision tree regression.

\section{Decision Forest Regression (DFR)}

It is an ensemble method that builds multiple decision trees and integrates their predicted models together to obtain a more accurate and stable model rather than depend on an individual tree [37], [38]. Each tree in the forest learns randomly from the samples of the training data. Some samples are selected to be used multiple times in an individual tree and some samples may not absolutely be selected as shown in Fig. 15. In other words, it is training each tree on different samples. Even though each tree may have a variance according to the training data, the forest will have minimum variance, but not at the value of increasing the bias [39]

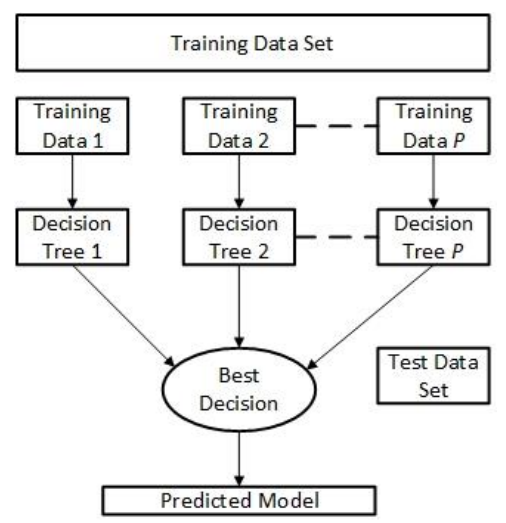

Fig. 15. Perspective of decision forest regression.

The used resampling method is bagging. In this method, a novel training data of $\dot{x}_{i}, \dot{y}_{i}{ }_{i=1}^{n}$ are randomly generated from the original training data of $x_{i}, y_{i}{ }_{i=1}^{n}$, and have the same number of samples as in the original training data, known as bootstrapping. In bagging, a predefined number set to $(|p|)$ of bootstrap samples $\dot{x}_{i}, \dot{y}_{i}{ }_{i=1}^{p}$ is generated according to [40], which shows that $63.2 \%$ of the original 
samples are reserved for a bootstrap sample. Hence, a decision tree algorithm is applied on each bootstrap sample of $\dot{x}_{i}, \dot{y}_{i}{ }_{i=1}^{p}$ in order to generate $|p|$ number of trees for the forest regression [41].

\section{TRAINING OF MACHINE LEARNING REGRESSIONS}

The first process in ML model development is the generation and collection of datasets for training and testing. There are three data sets generated for the proposed antenna, namely, simulation, measurement, and test [14], [15]. Simulation data are generated by CST Microwave Studio, while the measurement data are given from Rhode \& Schwarz ZVB20 Vector Network Analyzer. Test data are obtained outside (extrapolation) of the simulation and measurement to independently test the quality (accuracy and generalization capability) of the trained model. $x_{i}, y_{i}{ }_{i=1}^{n}$ could be used for training the ML methods and testing the resulting ML models. The number of training data is $n=5001$ samples and the test data are $\bar{m}=1001$ samples. The procedure for obtaining extrapolation test data is by calculating the average of first set of $s=5$ samples $s=i_{1}, \ldots, i_{5}$ of the training data to determine the first sample of the test data, and then this process will uniformly continue to last 5 samples $s=i_{4097}, \ldots, i_{5001}$. In general, average $\bar{m}$ of each $s$ set is obtained by

$$
\bar{m}=\frac{1}{s}\left(\sum_{i=1}^{s} x_{\min , i}+\ldots+x_{\max , s}\right),
$$

where $x_{\min , i}$ and $x_{\max , s}$ are the set of first and last values of the $s$ of $n$ samples in the training data. Each average of $\bar{m}_{1}, \bar{m}_{2}, \bar{m}_{3}, \ldots, \bar{m}_{1001}$ determines one sample of the test data. Note that $\bar{m}_{1}, \ldots, \bar{m}_{1001} \notin x_{i}, y_{i}{ }_{i}^{n}$. A sample generated is a uniform and independent from the training data, where samples between $x_{\max , s}$ and $x_{\min , i}$ are independent.

In training process, samples of the training data are being iteratively provided to the model. Then the model exercises the current parameter samples and predicts a new prediction. Prediction is compared to target, and the difference is shown as an error. Then returns to modulate and update itself to decrease that error in next prediction states. This means that model will update the values of its parameters according to the ML regression algorithms based on which they were generated as shown in Fig. 16. Models are trained by modulating their parameters' values to realize preferable results. Therefore, models are the results of the learned ML from training data. The measured model that is used as a target for prediction is seen in Fig. 11 and Fig. 12.

The dimensions and configuration parameters of RSMA are variables, hence inputs and outputs $\left\{x_{\mathrm{i}}, y_{\mathrm{i}}\right\}$ of the corresponding RSMA machine learning model are given by:

$$
x=\left[l_{1}, l_{2}, l_{3}, f, s_{11}^{s i m}\right]^{T}
$$

$$
y=S_{11}^{T},
$$

where $f$ is operating frequency, $l_{1}, l_{2}$, and $l_{3}$ represent the width, length of middle microstrip, and height of the radiating element, respectively. $S_{11}^{\text {sim }}$ is the simulation data. The subscript $T$ points out the transpose of the input and output vectors or matrices. $x_{i}, y_{i}{ }_{i}^{n}$ can be expressed based on RSMA modeling problem as shown in (7). According to the proposed study, there are one output variable and multiple input variables. Note that ML models can accommodate and learn from multiple input variables to predict multiple output variables.

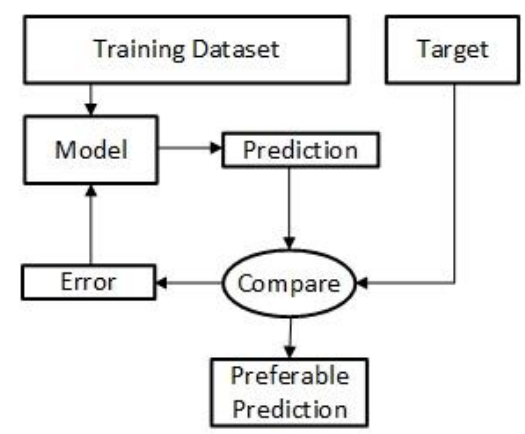

Fig. 16. Perspective of machine learning regression work.

The accuracy of the resulting regression models has been first achieved through the methods' plots as shown in Figs. 17-19, and then through measuring errors as shown in Table III. It is in line with using multiple predicting accuracy measures.

ANN model is developed using Levenberg-Marquardt learning algorithm that combines two minimization methods, which are gradient descent and Gauss-Newton, with a learning rate of 0.005 , number of iterations of 100 , initial learning weights diameter of 0.1 , and 1 hidden layer, including number of neurons of 100 (Fig. 17).

Decision tree regression model is developed using single parameter for the trainer mode, number of leaves/tree is maximized to 20, number of samples/leaf nodes is minimized to 10 , learning rate of 0.2 and the total number of trees constructed is 100 (Fig. 18).

Decision forest regression model is developed using number of decision trees of 8 , maximum depth of the decision trees of 32, number of random splits/nodes of 128 , and maximum number of samples/leaf nodes of 1 (Fig. 19).

The error obtained by different measures is compared using measured $\left(y^{\text {meas }}\right)$ and predicted $\left(y^{\text {pred }}\right)$ data. A measure $y^{\text {pred }}$ is realized for comparing errors with $y^{\text {meas }}$. The main result was that the DTR and DFR algorithms have the best measure of error as shown in Table III.

TABLE III. ACCURACY COMPARISON BETWEEN REGRESSION MODELS

\begin{tabular}{|c|c|c|c|c|}
\hline Regression model & MSE & RMSE & RAE & RSE \\
\hline ANN & 2.021 & 3.289 & 1.092 & 1.150 \\
\hline DTR & 0.038 & 0.491 & 0.021 & 0.025 \\
\hline DFR & 0.055 & 0.263 & 0.029 & 0.077 \\
\hline
\end{tabular}


Figure 20 shows the extent of the correlation between the measurement and the prediction for regression models. While the correlation in the Fig. 20(b) and Fig. 20(c) is a positive remarkable, the correlation in the Fig. 20(a) is negative and quite small.

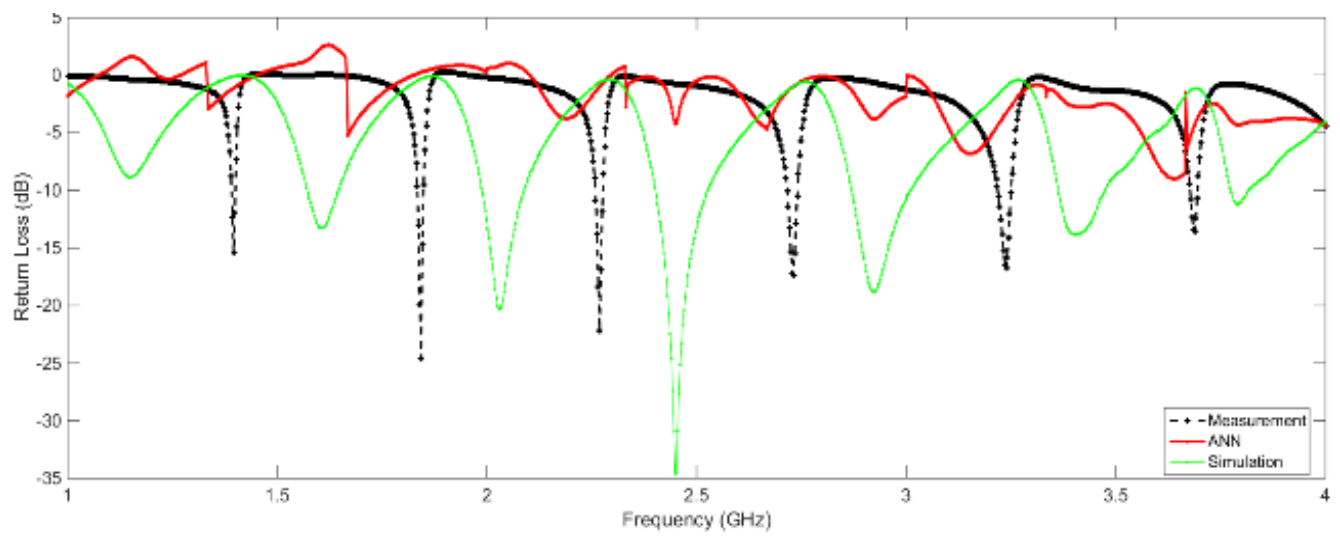

Fig. 17. Comparison of $S_{11}$ of measurement, simulation, and ANN prediction models.

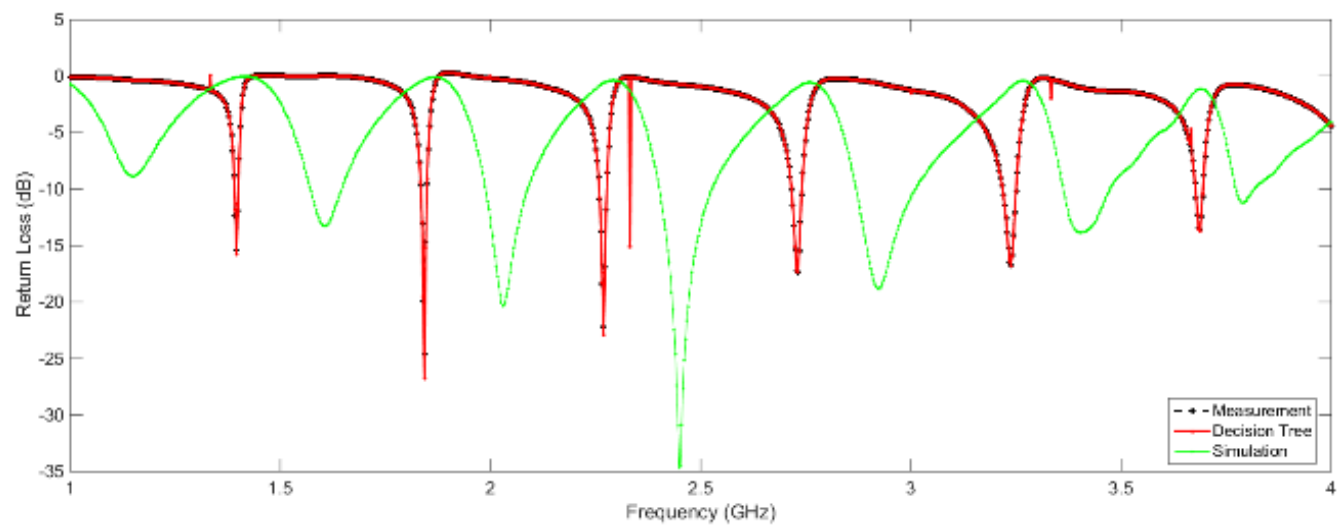

Fig. 18. Comparison of $S_{11}$ of measurement, simulation, and DTR prediction models.

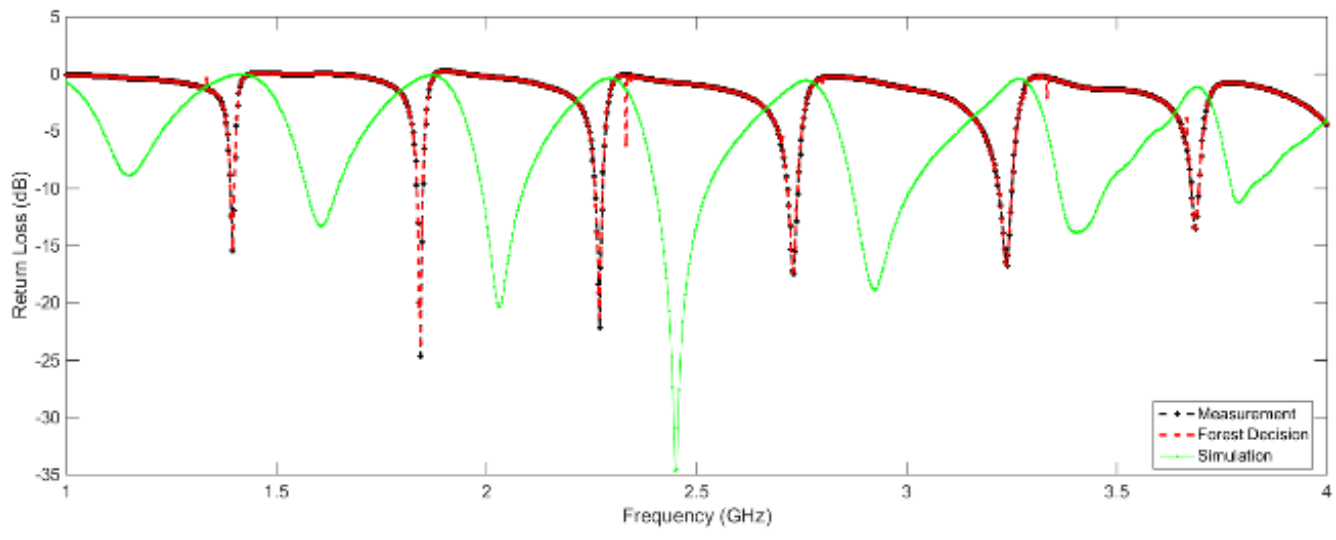

Fig. 19. Comparison of $S_{11}$ of measurement, simulation, and DFR prediction models.

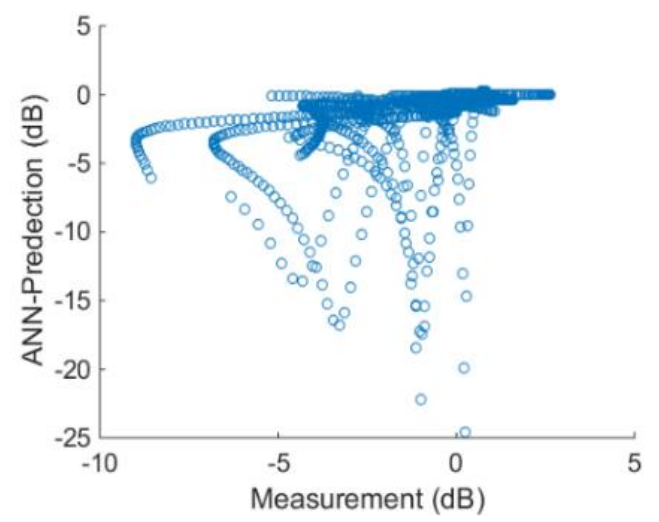

(a)

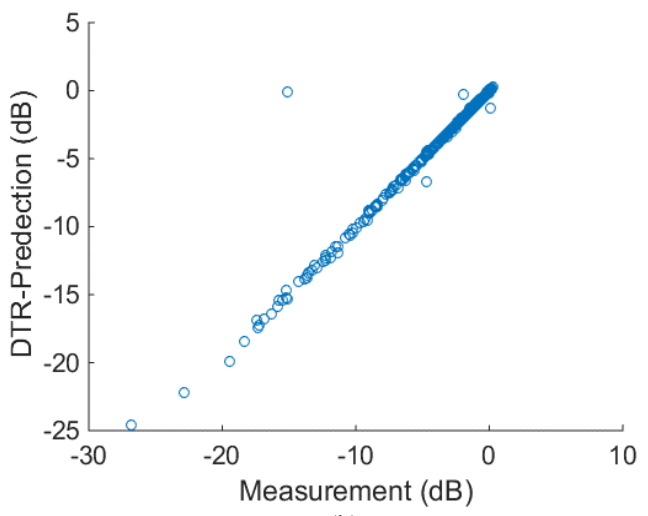

(b) 


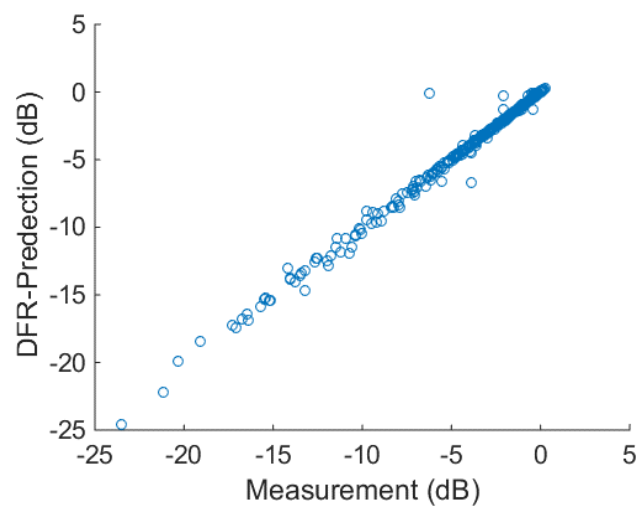

(c)

Fig. 20. Scatter graphs for a) ANN, b) DTR, and c) DFR.

\section{COMPARISON AND ANALYSIS RESULTS}

\section{A. Measurement and Simulation}

Figure 4 and Figure 12 show the results of S-parameters of the all simulation and measurement stages. Therefore, the change between resonating frequencies and bandwidth in each case is clearly appeared. There are shifting without exact overlapping and generating new operating frequencies in every simulation stage, in addition to displaying multiple resonant frequencies in the measurement case, which are $1.4 \mathrm{GHz}, \quad 1.8 \mathrm{GHz}, 2.27 \mathrm{GHz}, 2.73 \mathrm{GHz}, 3.26 \mathrm{GHz}$, and $3.73 \mathrm{GHz}$ as shown in Fig. 12 and Fig. 13. It depends on adding a new spiral arm in each case. $3.4 \mathrm{GHz}-3.8 \mathrm{GHz}$ band with the bandwidth of $200 \mathrm{MHz}$ is widely recognized as a supported band for 5G systems in LTE TDD mode, allocating asymmetric distribution of uplink and downlink resources in wireless systems. It is clearly shown that the number of resonant frequencies can be multiplied by increasing the length of the spiral conductors $(C)$, with considering the empty space between them. The bandwidth is wider in the first, second, third, and fourth simulation cases than in the measurement case, but the value of the return loss in measurement case is less than in simulation cases. Equations (2), (5), and (6) theoretically show approximate results agreeing fairly with simulation results in some cases, while the results do not agree in most simulations (see Table II and the section of first and fourth configurations). The far-field patterns show the radiated power as a function of the direction of z-axis and vary as a function of the angles of $\varnothing=0^{\circ}$ and $\varnothing=90^{\circ}$. Observing two-dimensional far-field patterns, the radiation is maximum at $0^{\circ}$ and $90^{\circ}$ along $\mathrm{z}$-axis and is a minimum broadside to the antenna. The angle HPBW is around $45 \%$ of the peak power. Remarkably, good patterns can be obtained with combining microstrip and spiral conductors.

\section{B. Measurement and Regression Methods}

As it can be observed from the regression models, the proposed DTR and DFR evidently realized accurate models when compared to ANN model. Compared to ANN, the high performance of DTR and DFR is mainly achieved from the systematic, non-parametric, and methodical feature of the tree structure that can predict the target variable through simple processes learned from measured training data. Thus, there is a remarkable agreement between the prediction and measurement models with some differences as shown in Fig. 18, Fig. 19, Fig. 20(b), and Fig. 20(c), as well as in Table III. While ANN model is not highly accurate, it hardly remains within measurement boundaries as shown in Fig. 17 and Fig. 20(a). For a straightforward comparison, Table III includes error metrics for used regression models that enhance understanding accuracy and generalization capability of the model of RSMA.

In [17] and [18], a similar comparative study is presented using three ML and EM models. As a result, ML techniques used in our study, [17], and [18] can additionally be utilized to recognize and solve significantly more complex antenna problems. Therefore, the results received from the studies imply that ML methods can be a parallel solution to EM simulation in novel antenna technology.

The analysis of the previous results may lead the researchers to focus on developing and optimizing antennas through artificial intelligence methods.

\section{CONCLUSIONS}

This article gradually discussed how to combine rectangular microstrip antennas with spiral antennas. The model results are numerical, 3D-EM, machine learning simulation, and measurement. In the sequence of simulation cases, the combined configuration called "spiral-shaped microstrip antenna" (RSMA) has been developed, making the goal of the proposed antenna, such as small size, good patterns, and less HPBW, to be achieved. The operating frequency range covers the range of $1 \mathrm{GHz}-4 \mathrm{GHz}$ (L-band and S-band). To verify the design of the proposed antenna, the model has been fabricated and measured. It can be a good choice for small covering with high data rate capacity, in addition to be operable and suitable for different wireless communication in indoor/outdoor environments (WLAN: $2.4 \mathrm{GHz}-2.48 \mathrm{GHz}$, WiMAX: $\quad 3.4 \mathrm{GHz}-3.69 \mathrm{GHz}$, and WiFi: $2.40 \mathrm{GHz}-2.48 \mathrm{GHz}$ ).

This research also explores three regression algorithms based on machine learning for predicting models of the proposed antenna, calculating the accuracy and generalization capability. Algorithms, such as decision tree regression, decision forest regression, and artificial neural network, were used in solving such modeling problem. Regression models predicted rightly with some acceptable 
differences. This work is successfully applied by regression algorithms for modeling the proposed antenna. The results indicate the reliability of the proposed prediction methods. Moreover, both researchers and practitioners may use different machine learning methods for modeling antennas.

\section{CONFLICTS OF INTEREST}

The author declares that he has no conflicts of interest.

\section{REFERENCES}

[1] Q. Wu, Y. Cao, H. Wang, and W. Hong, "Machine-learning-assisted optimization and its application to antenna designs: Opportunities and challenges", China Communications, vol. 17, no. 4, pp. 152-164, Apr. 2020. DOI: 10.23919/JCC.2020.04.014

[2] A. Kumar, F.-Y. Meng, C. Wang, K. K. Adhikari, T. Qiang, Q. Wu, and $\mathrm{Y} . \mathrm{Wu}$, "Design analysis of integrated passive device-based balun devices with high selectivity for mobile application", IEEE Access, vol. 7, pp. 23169-23176, 2019. DOI 10.1109/ACCESS.2019.2898513

[3] Ch.-N. Chiu and W.-H. Chuang, "A novel dual-band spiral antenna for a satellite and terrestrial communication system", IEEE Antennas and Wireless Propagation Letters, vol. 8, pp. 624-626, 2009. DOI: 10.1109/LAWP.2009.2021283.

[4] A. Aoad and M. S. U. Türeli, "Design, simulation, and fabrication of a small size of a new spiral shaped of circular microstrip patch antenna", Microwave and Optical Technology Letters, vol. 60, no. 12, pp. 2912-2918, 2018. DOI: 10.1002/mop.31444.

[5] T. A. Milligan, Modern Antenna Design. John Wiley \& Sons, Inc., Publication, 2005. DOI: 10.1002/0471720615.

[6] J. Meiguni and D. Pommerenke, "Theory and experiment of UWB Archimedean conformal spiral antennas", IEEE Transactions on Antennas and Propagation, vol. 67, no. 10, pp. 6371-6377, 2019. DOI: $10.1109 /$ TAP.2019.2925183.

[7] A. Sohrabi, H. Dashti, and J. Ahmadi-Shokouh, "Design and analysis of a broadband electrically small antenna using characteristic mode theory", International Journal of Electronics and Communications, vol. 113, pp. 1-8, 2020. DOI: 10.1016/j.aeue.2019.152991.

[8] Y. Liu, H. Wang, K. Li, and Sh. Gong, "RCS reduction of a patch array antenna based on microstrip resonators", IEEE Antennas and Wireless Propagation Letters, vol. 14, pp. 4-7, 2015. DOI: 10.1109/LAWP.2014.2354341.

[9] H. Steyskal, J. Ramprecht, and H. Holter, "Spiral elements for broadband phased arrays", IEEE Transactions on Antennas and Propagation, vol. 53, no. 8, pp. 2558-2562, 2005. DOI: 10.1109/TAP.2005.852309.

[10] N. A. Aboserwal, J. L. Salazar, J. A. Ortiz, J. D. Díaz, C. Fulton, and R. D. Palmer, "Source current polarization impact on the crosspolarization definition of practical antenna elements: Theory and applications", IEEE Transactions on Antennas and Propagation, vol. 66, no. 9, pp. 4391-4406, 2018. DOI: 10.1109/TAP.2018.2845945.

[11] E. Gschwendtner, D. Loffler, and W. Wiesbeck, "Spiral antenna with external feeding for planar applications", in Proc. of 1999 IEEE Africon. 5th Africon Conference in Africa, 1999, pp. 1011-1014, vol. 2. DOI: $10.1109 / A F R C O N .1999 .821910$.

[12] B. Liu, H. Aliakbarian, Zh. Ma, G. A. E. Vandenbosch, G. Gielen, and P. Excell, "An efficient method for antenna design optimization based on evolutionary computation and machine learning techniques", IEEE Transactions on Antennas and Propagation, vol. 62, no. 1, pp. 7-18, 2014. DOI: 10.1109/TAP.2013.2283605.

[13] B. Liu, M. O. Akinsolu, N. Ali, and R. Abd-Alhameed, "Efficient global optimisation of microwave antennas based on a parallel surrogate model-assisted evolutionary algorithm", IET Microwaves, Antennas \& Propagation, vol. 13, no. 2, pp. 149-155, 2019. DOI: 10.1049/iet-map.2018.5009. DOI: 10.1049/iet-map.2018.5009.

[14] A. Aoad, M. Simsek, and Z. Aydin, "Design of a reconfigurable 5fingers shaped microstrip patch antenna by artificial neural networks", International Journal of Advanced Research in Computer Science and Software Engineering (IJARCSSE), vol. 4, no. 10, pp. 61-70, 2014.

[15] A. Aoad, M. Simsek and Z. Aydin, "Knowledge based response correction method for design of reconfigurable N-shaped microstrip patch antenna using inverse ANNs", International Journal of Numerical Modelling: Electronic Networks, Devices and Fields, vol. 30, nos. 3-4, p. e2129, 2017. DOI: 10.1002/jnm.2129.

[16] M. Simsek and A. Aoad, "Multiple operating frequency selections for reconfigurable antenna design by SM based optimisation", IET
Microwaves, Antennas \& Propagation, vol. 11, no. 13, pp. 18981908, 2017. DOI: 10.1049/iet-map.2017.0273.

[17] Y. Sharma, H. H. Zhang, and H. Xin, "Machine learning techniques for optimizing design of double T-shaped monopole antenna", IEEE Transactions on Antennas and Propagation, vol. 68, no. 7, pp. 56585663, 2020. DOI: 10.1109/TAP.2020.2966051.

[18] Q. Wu, H. Wang, and W. Hong, "Multistage collaborative machine learning and its application to antenna modeling and optimization", IEEE Transactions on Antennas and Propagation, vol. 68, no. 5, pp. 3397-3409, 2020. DOI: 10.1109/TAP.2019.2963570.

[19] W. Y. Al-Rashdan and A. Tahat, "A comparative performance evaluation of machine learning algorithms for fingerprinting based localization in DM-MIMO wireless systems relying on big data techniques", IEEE Access, vol. 8, pp. 109522-109534, 2020. DOI: 10.1109/ACCESS.2020.3001912.

[20] A. M. Alzahed, S. M. Mikki, and Y. M. M. Antar, "Nonlinear mutual coupling compensation operator design using a novel electromagnetic machine learning paradigm", IEEE Antennas and Wireless Propagation Letters, vol. 18, no. 5, pp. 861-865, 2019. DOI: 10.1109/LAWP.2019.2903787.

[21] J.-M. Lu, Y.-Sh. Li, and M.-Sh. Zhang, "An efficient SPICEcompatible cavity resonant model for microstrip lines", IEEE Transactions on Components, Packaging and Manufacturing Technology, vol. 1, no. 4, pp. 574-585, 2011. DOI: 10.1109/TCPMT.2010.2099714.

[22] R. Berro, S. Bories, and Ch. Delaveaud, "Transmission line model for lossy inverted F antenna miniaturization", in Proc. of 2019 International Conference on Electromagnetics in Advanced Applications (ICEAA), Granada, Spain, 2019, pp. 0797-0802. DOI: 10.1109/ICEAA.2019.8879094.

[23] I. J. Bahl and P. Bhartia, Microstrip Antennas. Dedham, MA: Artech house, 1980 .

[24] N. Ma and H. Zhao, "Reduction of the mutual coupling between aperture coupled microstrip patch antennas using EBG structure", in Proc. of 2014 IEEE International Wireless Symposium, X'ian, China, 2014, pp. 1-4. DOI: 10.1109/IEEE-IWS.2014.6864201.

[25] Sh. Lu, H. T. Hui, and M. Bialkowski, "Optimizing MIMO channel capacities under the influence of antenna mutual coupling", IEEE Antennas and Wireless Propagation Letters, vol. 7, pp. 287-290, 2008. DOI: 10.1109/LAWP.2008.928474.

[26] L. Sevgi and G. Çakir, "A broad array of Archimedean spiral antennas for wireless applications", Microwave and Optical Technology Letters, vol. 48, no. 1, pp. 195-200, 2006. DOI: 10.1002/mop.21304.

[27] B.-L. Ooi, D.-X. Xu, P.-Sh. Kooi, and F.-j. Lin, "An improved prediction of series resistance in spiral inductor modeling with eddycurrent effect", IEEE Transactions on Microwave Theory and Techniques, vol. 50, no. 9, pp. 2202-2206, 2002. DOI: 10.1109/TMTT.2002.802337.

[28] H. Greenhouse, "Design of planar rectangular microelectronic inductors", IEEE Transactions on Parts, Hybrids, and Packaging, vol. 10, no. 2, pp. 101-109, 1974. DOI: 10.1109/TPHP.1974.1134841.

[29] T. Itoh, Numerical Techniques for Microwave and Millimeter-Wave Passive Structures. New York: John Wiley, 1989. DOI: $10.1109 / 20.34328$.

[30] T. M. Mitchell, Machine Learning. The McGraw-Hill Companies, Inc., 1997.

[31] Ch.-Y. Wang and M.-Y. Lin, "Prediction of accrual expenses in balance sheet using decision trees and linear regression", in Proc. of 2016 Conference on Technologies and Applications of Artificial Intelligence (TAAI), Hsinchu, 2016, pp. 73-77. DOI: 10.1109/TAAI.2016.7880157.

[32] M. Collier and R. Shahan, Fundamentals of Azure. Redmond, Washington: Microsoft Press, 2015.

[33] S. Preston, Microsoft Azure Terminology and Concepts. Apress, 2016 DOI: 10.1007/978-1-4842-1476-3_2.

[34] R. San Millán-Castillo, E. Morgado, and R. Goya-Esteban, "On the use of decision tree regression for predicting vibration frequency response of handheld probes", IEEE Sensors Journal, vol. 20, no. 8, pp. 4120-4130, 2020. DOI: 10.1109/JSEN.2019.2962497.

[35] $\mathrm{Ph}$. A. Chou, "Optimal partitioning for classification and regression trees", IEEE Transactions on Pattern Analysis and Machine Intelligence, vol. 13, no. 4, pp. 340-354, Apr. 1991. DOI: $10.1109 / 34.88569$.

[36] S. Danwa, H. Ning, and L. Dandan, "Construction of forestry resource classification rule decision tree based on ID3 algorithm", in Proc. of 2009 First International Workshop on Education Technology and Computer Science, Wuhan, Hubei, China, 2009, pp. 867-870. DOI: 
10.1109/ETCS.2009.730.

[37] M. Chigullapally and S. Patnaik, "Wi-Fi $802.11 \mathrm{mc}$ distance classification and error reduction using machine learning", in Proc. of the 5th International Conference on Communication and Electronics Systems (ICCES 2020), Coimbatore, India, 2020, pp. 599-604. DOI: 10.1109/ICCES48766.2020.9137922.

[38] L. Rokach, "Decision forest: Twenty years of research", Information Fusion, vol. 27, pp. 111-125, 2016. DOI: 10.1016/j.inffus.2015.06.005.
[39] M. N. Adnan and M. Zahidul Islam, "Forest PA: Constructing a decision forest by penalizing attributes used in previous trees", Expert Systems with Applications, vol. 89, pp. 389-403, 2017. DOI: 10.1016/j.eswa.2017.08.002.

[40] J. Han, J. Pei, and M. Kamber, Data Mining: Concepts and Techniques. Morgan Kaufmann Publishers, 2006.

[41] L. Breiman, J. Friedman, R. Olshen, and R. Stone, "Classification and regression trees", in Regression Trees. CRC press, 2017, pp. 216-265. DOI: $10.1201 / 9781315139470-8$.

This article is an open access article distributed under the terms and conditions of the Creative Commons Attribution 4.0 (CC BY 4.0) license (http://creativecommons.org/licenses/by/4.0/). 